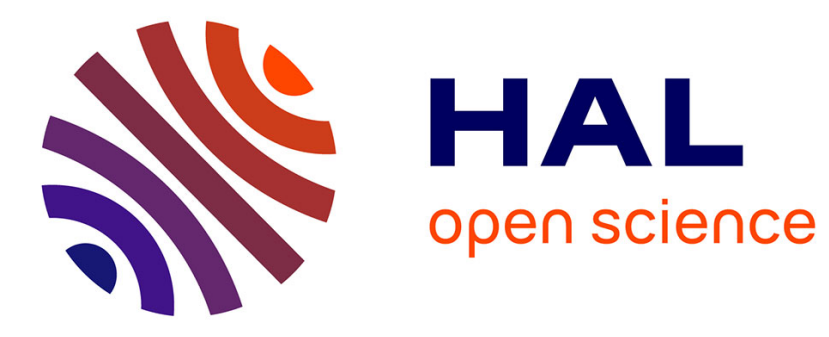

\title{
Comment sortir de la dichotomie technique/société
}

\author{
Madeleine Akrich
}

\section{To cite this version:}

Madeleine Akrich. Comment sortir de la dichotomie technique/société: Présentation des diverses sociologies de la technique. Latour, B. et Lemonnier, P. De la préhistoire aux missiles balistiques: l'intelligence sociale des techniques, La Découverte, pp.105-131, 1994. halshs-00081733

\section{HAL Id: halshs-00081733 \\ https://shs.hal.science/halshs-00081733}

Submitted on 3 Jul 2006

HAL is a multi-disciplinary open access archive for the deposit and dissemination of scientific research documents, whether they are published or not. The documents may come from teaching and research institutions in France or abroad, or from public or private research centers.
L'archive ouverte pluridisciplinaire HAL, est destinée au dépôt et à la diffusion de documents scientifiques de niveau recherche, publiés ou non, émanant des établissements d'enseignement et de recherche français ou étrangers, des laboratoires publics ou privés. 
Akrich, M., 1994, Comment sortir de la dichotomie technique/société. Présentation des diverses sociologies de la technique, In Latour, B.et Lemonnier, P., ed, De la préhistoire aux missiles balistiques : l'intelligence sociale des techniques, Paris, La Découverte, pp.105-131.

\title{
DE LA SOCIOLOGIE DES TECHNIQUES A LA SOCIOLOGIE... \\ ou comment sortir de la dichotomie: \\ genèse sociale des techniques/ genèse technique des hommes
}

\author{
Madeleine Akrich \\ CSI-ENSMP
}

Bien que la question des techniques ait depuis longtemps suscité réflexions, commentaires, analyses de la part des chercheurs en sciences sociales, la sociologie des techniques, qui, sous son acception restreinte, est de création récente ${ }^{1}$, n'apparaît pas aujourd'hui comme un champ de recherches aussi fortement structuré que celui de la sociologie des sciences. A l'image des techniques elles-mêmes, qui se prêtent à de multiples définitions, les angles d'attaque adoptés sont des plus divers, de la philosophie, à l'histoire en passant par l'économie, la sociologie ou l'anthropologie; notons que, par ailleurs, l'étanchéité disciplinaire n'est pas aussi forte que dans d'autres domaines: de plus en plus fréquemment, des sociologues s'appuient sur les travaux des historiens, des économistes citent ceux des sociologues, et vice-versa. Face à cette mosaïque de travaux qui rend vaine toute prétention à l'exhaustivité, nous avons pris le parti d'axer notre présentation autour d'un nombre restreint d'auteurs, choisis avec un double objectif: appartenant à des disciplines variées, leurs travaux donnent un aperçu des contributions de chacune d'entre elles; par ailleurs, leurs pensées sont suffisamment contrastées pour offrir un panorama aussi large que possible des thématiques, des problèmes posés, des débats auxquels ils ont donné lieu et des réponses qui ont été apportées, ce qui doit permettre de donner des points de repère par rapport auxquels situer un grand nombre d'autres travaux.

En première approximation, deux pôles peuvent être distingués dans cette masse de littérature: d'un côté, on trouve tout un ensemble de recherches - historiques, économiques ou sociologiques - qui se sont attachées au problème de l'émergence des techniques; il s'agit de comprendre pourquoi certains acteurs éprouvent à un moment donné le besoin d'innover et de rendre compte de la forme prise par ces innovations, d'expliquer leur succès ou leur échec, d'analyser leurs effets économiques et/ou sociaux. De l'autre côté, la

\footnotetext{
${ }^{1}$ voir par exemple BIJKER, HUGHES and PINCH (1989) et MACKENZIE and WAJCMAN (1985)
} 
question n'est plus tant de savoir ce qui fait les techniques mais plutôt de caractériser ce qu'elles font: on trouve ici un ensemble de modèles à tendance philosophique ou anthropologique qui visent une compréhension globale du phénomène humain ou social et dans lesquels les techniques occupent une place centrale - à côté d'autres auteurs, Marx, Mumford, Leroi-Gourhan constituent de bons exemples de cette approche qui se préoccupe assez peu des modalités concrètes par lesquelles les techniques voient le jour. Notons que les développements récents de l'économie et de la sociologie des techniques sont en train d'opérer un rapprochement entre ces deux pôles; s'appuyant sur les acquis de la sociologie des sciences, ils partent en effet d'une hypothèse méthodologique qui peut être formulée de la façon suivante: pour comprendre ce que sont et ce que font les techniques, il est nécessaire de les déconstruire et d'analyser leur constitution, ou, autrement dit, le processus d'innovation et les controverses auxquelles il donne lieu mettent en jeu non seulement la définition des techniques, mais, corrélativement et de façon indissociable, celle de la société dans laquelle s'insèreront les techniques.

On pourrait avoir tendance à schématiser cette bipolarisation des travaux en une partition entre deux courants, le premier qui relèverait d'une construction sociale de la technologie et le second d'une construction technique de la société ${ }^{2}$, et même à supposer que leurs modes explicatifs peuvent être décrits par l'opposition déterminisme social/ déterminisme technique. La sociologie récente des techniques, qui s'est construite en partie sur la réfutation conjointe de ces deux thèses opposées, en a peut-être exagéré la portée: une analyse attentive des textes révèle que rares, peut-être même inexistants, sont les auteurs qui tombent sous le coup de l'une ou l'autre de ces accusations. Les tenants les plus forcenés d'une construction sociale des techniques sont toujours à un moment donné obligés de tenir compte de la résistance des objets, ou en d'autres termes, de facteurs techniques à côté des facteurs sociaux, de même que, symétriquement, les déterministes techniques admettent, ne seraitce que sous la forme de l'historicité des techniques, l'existence de contraintes sociales, économiques ou démographiques. A ce découpage qui tranche sans égards dans le vif, nous préférons substituer une gradation progressive des modèles, qui se distinguent par leur plus ou moins grande endogénéisation de tel ou tel type de facteurs: ainsi par exemple, ce n'est pas que les prétendus déterministes techniques ignorent le social, c'est plutôt qu'ils choisissent de le laisser en partie à la frontière de leurs modèles et de s'y intéresser davantage en tant que résultat du processus technique qu'en tant que cause de ce même processus. Dans ce schéma, la sociologie récente des techniques tend vers l'un des points extrêmes, en se donnant comme objectif l'explicitation des processus par lesquels se construisent conjointement la technique et le social.

La bipolarité que nous avons relevée plus haut doit donc s'interpréter comme le résultat de I'histoire des disciplines, de leurs interrogations et de la manière dont elles se sont constituées et non comme une opposition théorique entre deux modalités explicatives inconciliables. Si l'on abandonne cette opposition trop commode entre déterminisme technique et déterminisme social, comment ordonner et décrire des pensées qui n'entrent dans aucune construction généalogique commune? Nous avons construit un certain nombre

\footnotetext{
${ }^{2}$ voir par exemple l'introduction de Mackenzie dans MACKENZIE and WAJCMAN (1985)
} 
de modèles contrastés à partir d'une grille de questions analogue à celle utilisée par Michel Callon dans sa présentation de la sociologie des sciences ${ }^{3}$, à savoir: a) comment les produits de l'activité technique sont-ils définis (dispositifs, savoirs, know-how, organisation sociale, mixte socio-technique...)?, b) quels sont les acteurs considérés (chercheurs, ingénieurs et techniciens, firmes, utilisateurs, marché, groupes sociaux, dispositifs techniques...)?, c) quel est le moteur de l'activité et du changement techniques?, d) à quelles conditions arrive-t-on à l'accord des différents acteurs impliqués et à la stabilisation des dispositifs techniques?, e) quelle est l'organisation sociale requise? f) comment l'activité technique elle-même est-elle catégorisée (ce qui, selon les cas, revient à s'interroger sur ce que font les techniques ou sur ce qui les fait)?

Trois groupes de modèles peuvent être distingués, selon la réponse qu'ils donnent à notre première question:

1) le groupe de "l'autonomie de la technique": dans ces modèles, la technique apparaît gouvernée de l'intérieur par des contraintes fortes qui déterminent son évolution de manière plus ou moins autonome par rapport à l'évolution économique, sociale ou politique.

2) le groupe "Construction sociale de la technique/Construction technique de la société" qui est caractérisé par une séparation entre deux entités, la technique et la société, qui interagissent et se co-déterminent.

3) le groupe "Seamless Web"4: il n'est plus possible ici de penser la technique hors de la société ou la société hors de la technique; l'une et l'autre émergent conjointement des processus d'innovation et la technique n'apparaît plus que comme une modalité particulière d'association durable des humains entre eux et avec des entités non-humaines.

\section{"AUTONOMOUS TECHNOLOGY"5 OU LA TECHNIQUE COMME SYSTĖME}

Comme nous venons de le souligner, les auteurs regroupés ici partagent une même hypothèse fondamentale pour le fonctionnement de leurs modèles, à savoir que la technique autodétermine, au moins pour une part significative, son propre développement. Nous distinguerons deux sous-ensembles de travaux selon l'intensité, "faible" ou "forte", de cette autodétermination.

\section{DE L'AUTONOMIE DES TECHNIQUES...}

L'oeuvre de Bertrand Gille représente une expression particulièrement aboutie du premier groupe, défini par une autodétermination partielle de la technique; cherchant à retracer sur une longue période l'histoire des techniques, il est confronté aux problèmes classiques de

${ }^{3}$ CALLON (1993)

${ }^{4}$ ou du "tissu sans coutures", selon l'expression devenue célèbre de HUGHES (1983).

${ }^{5}$ Nous reprenons ici le titre anglais du livre de WINNER (1983). 
I'historien - comment ordonner des masses conséquentes de données et comment définir une périodisation? -, problèmes qu'il résoud par l'introduction d'un certain nombre de concepts à la fois descriptifs et explicatifs. L'ensemble technique constitue le point nodal de son modèle: il est défini comme l'ensemble des techniques (elles-mêmes constituées de dispositifs, de savoirs et de savoir-faire) mobilisées dans un acte particulier: la fabrication de la fonte exige des hauts-fourneaux et des connaissances quant à leur conduite et leur contrôle, des dispositifs de production d'énergie etc. A partir de là, l'on peut soit descendre dans le détail jusqu'aux structures techniques qui correspondent aux plus petites unités isolables dans un ensemble technique, soit remonter la filière technique qui est constituée de l'ensemble des ensembles techniques présupposés par un ensemble technique particulier: ainsi, l'ensemble technique défini par la fabrication de la fonte repose sur l'existence d'autres ensembles techniques qui concernent l'extraction des minerais et leur transport, la fabrication des hauts-fourneaux, etc. Cette construction théorique implique de fait des interrelations fortes entre tous les ensembles techniques, que ce soit verticalement au travers des filières, ou horizontalement par le biais des structures techniques qui peuvent être incorporées dans de nombreux ensembles techniques. Ce qui amène Gille à postuler l'existence d'un système technique autonome, défini comme l'ensemble cohérent de toutes les techniques utilisées à une époque donnée. L'évolution du système technique est conditionnée à la fois par les techniques elles-mêmes dans lesquelles sont inscrites dès le départ certaines limites de développement (taille, puissance, rendement etc.), et par l'exigence de cohérence entre les ensembles techniques associés dans les filières. La direction et le rythme de cette évolution est cependant commandée de l'extérieur du système technique, au travers de l'expression des besoins par laquelle s'effectue l'articulation entre le système technique et les autres systèmes postulés par Gille, système politique, système économique, système social, système démographique, qui, chacun, obéissent à la même contrainte de cohérence interne que le système technique. Lorsqu'il y a cohérence entre ces différents systèmes et le système technique, c'est-à-dire lorsque les besoins exprimés peuvent être satisfaits moyennant une transformation des techniques existantes qui ne remet pas en cause la cohérence interne du système technique, l'on se trouve en régime stable. En revanche, lorsque les limites inhérentes aux techniques sont atteintes et rendent impossible leur adaptation à de nouveaux besoins, une crise apparaît qui ne peut être résolue que par l'émergence d'un nouveau système technique s'organisant autour d'une technologie centrale (la machine à vapeur au 19ème siècle, l'électronique aujourd'hui...). Dans tous les cas, l'évolution des techniques se fait soit par transfert de structures techniques d'une filière à une autre, soit par application des connaissances scientifiques: le décalage éventuel entre le stock de connaissances disponibles et celui qui est nécessaire pour passer d'un système technique à un autre permet à Gille d'expliquer la plus ou moins longue durée des crises. Bien que les crises soient en quelque sorte "programmées" dans la technologie elle-même, ce qui a parfois fait, à tort, considérer Gille comme un déterministe technique, elles ne peuvent être amorcées sans un détonateur extérieur au système technique. Nous nous trouvons donc en face d'un modèle à double causalité qui ménage une articulation entre les phénomènes techniques et les phénomènes sociaux au sens large, articulation que l'on retrouve chez certains philosophes de la technique comme Lafitte ou Simondon: chez ces deux auteurs, l'on observe la même tentative de fonder une science des objets techniques qui s'appuie sur l'élucidation des règles de leur évolution. Dans les deux cas, le milieu ou l'environnement, qui comprend tout ce qui n'est pas strictement technique, 
joue un rôle crucial sur les directions prises par l'évolution des techniques, elle-même régie par des règles internes qui déterminent le champ des possibles.

\section{... À LA DOMINATION DES TECHNIQUES}

Dans les modèles à "forte autodétermination", au contraire, cette double causalité est sinon absente du moins rigoureusement circonscrite. C'est aussi qu'il ne s'agit plus de comprendre comment les techniques se développent et évoluent, mais plutôt de montrer, et de dénoncer, l'impérialisme technique qui caractérise les sociétés modernes. Dans les travaux de Marcuse comme dans ceux d'Ellul, on assiste à un même double mouvement d'autonomisation des techniques, au sens où elles se mettent à proliférer et à se développer par l'effet de leur propre logique, et de colonisation progressive de tous les domaines d'activité humaine par les techniques... Au point d'en arriver, chez Marcuse, à l'unidimensionnalité, c'est-à-dire à l'indifférenciation des ordres antérieurement maintenus séparés (politique, économie, prestige etc.) rabattus sur la seule logique technique, et chez Ellul à l'instauration du système technicien marqué par la prééminence d'une seule forme de médiation entre les hommes eux-mêmes et avec leur environnement, la médiation technique. Bien que ces deux auteurs aboutissent à des systèmes comparables, ils n'admettent pas tout à fait les mêmes points de départ: Marcuse se situe dans la mouvance marxiste et à ce titre, l'extension toujours plus grande de la rationalité dans le système de production, extension dont la finalité première est l'asservissement de l'ouvrier au capitaliste, constitue le moteur initial de l'évolution: de son point de vue, la technique se présente comme une manière d'obtenir la cohésion des forces sociales et d'utiliser la nature en support de domination. Pour Ellul, il n'y a pas à chercher autre chose derrière la technique qu'elle-même: la forme de rationalité, qui est inhérente à son développement, porte en elle-même l'exigence d'une performance, d'un "progrès", d'une cohérence toujours accrus qui ne peuvent se réaliser que par annexion progressive de tous les champs d'activité humaine. La portée de ces différences ne doit cependant pas être exagérée: très rapidement, chez Marcuse, le capitaliste devient fantômatique et n'est plus incarné que dans la technique elle-même qui poursuit son évolution autonome et inexorable.

WINNER OU COMMENT PASSER DE LA DOMINATION DES TECHNIQUES À LA CONSTRUCTION SOCIALE DES TECHNIQUES

Les travaux de Langdon Winner, abondamment cités par différents courants de recherche, sont difficilement classables, car ils ménagent plusieurs modalités d'action des techniques. S'il s'agit toujours, comme chez Marcuse et Ellul, de contester le caractère totalitaire de la technologie, la condamnation est plus nuancée: Winner introduit une distinction entre les "inherently political technologies", dont le nucléaire est l'archétype, et qui supposent pour fonctionner la mise en place d'un Etat centralisé et policier, et les autres technologies dites "flexibles", emblématisées par le solaire, qui se prêtent à toutes sortes de configurations socio-politiques, y compris l'autogestion et/ou la décentralisation. Notons que, par ailleurs, certains groupes sociaux, qu'ils soient réactionnaires ou progressistes, peuvent donner à des technologies flexibles une forme qui renforce la ségrégation et les inégalités existantes ou au contraire, permette l'expression démocratique, idée qui a permis de rapprocher Winner du courant constructiviste. Pour Winner, si on laisse se développer les "inherently political 
technologies", on passe à un système dans lequel l'évolution sociale et politique est commandée par la technique, ce qui comme chez Marcuse ou Ellul, conduit à l'indifférenciation de sphères d'activité (travail, vie privée, organisation politique...) originairement séparées; d'où l'importance de l'évaluation sociale des techniques qui, seule, permet de sauver la démocratie des périls technologiques qui la guettent.

\section{CONCLUSION}

Au delà de la distinction que nous avons opérée entre les modèles à autodétermination "faible" et "forte", et à l'exception de Winner qui présente un modèle plus ambigu que les autres auteurs, plusieurs points de convergence nous paraissent devoir être notés.

D'une part, les individus, et en particulier ceux qui sont à l'origine des innovations, n'ont pratiquement pas de place en tant qu'acteurs véritables dans ces modèles: que la paternité de tel ou tel dispositif technique soit attribuée à un individu particulier est affaire de circonstances et ne représente qu'un phénomène contingent par rapport à l'ensemble des déterminants véritables de l'évolution technique; à partir du moment où certaines conditions, propres à chaque modèle, sont réunies, il se trouvera toujours un ou plusieurs agents humains pour donner le coup de pouce nécessaire. La technique, qui, comme nous l'avons vu, recouvre des réalités différentes selon les auteurs, est toujours un acteur prédominant.

En second lieu, les différents auteurs que nous avons envisagés s'appuient de façon explicite sur une logique de système, ce qui revient à dire que: 1) l'ensemble des entités mobilisées par chaque modèle sont interreliées - ce qui implique en particulier qu'il n'y a pas de différence fondamentale entre les phénomènes microscopiques et macroscopiques; 2) la stabilisation et/ou l'évolution de ces systèmes sont commandées par la même exigence, à savoir la mise en cohérence des différents composants des systèmes.

Enfin, on notera que ni I'"application" des connaissances scientifiques au développement technique, ni la détermination des besoins et leur traduction en dispositifs techniques ne sont problématiques pour les différents auteurs analysés: la transparence accordée à ces opérations contraste, comme on le verra dans la suite, avec les efforts de la sociologie des techniques actuelle qui consacre une bonne part de son travail à l'analyse de ces opérations considérées comme cruciales dans le développement des techniques.

\section{CONSTRUCTION SOCIALE DES TECHNIQUES/CONSTRUCTION TECHNIQUE DE LA SOCIÉTÉ OU LA TENDANCE ÉVOLUTIONNISTE}

Les travaux regroupés dans cette partie s'attachent à l'analyse des modalités d'interaction entre techniques et sociétés, entités qui restent plutôt séparées l'une de l'autre. Deux sousensembles de modèles assez différents peuvent être distingués, selon que le moteur du changement technique (question c)) est défini de façon générale ou au contraire, ne peut s'appréhender que localement. Le premier groupe comprend des modèles très hétérogènes, qui ont cependant en commun une portée globalisante: ils couvrent de longues périodes et se donnent pour objectif d'expliquer l'ensemble de l'évolution sociale, technique, politique et 
économique. Le second groupe est plus homogène et rassemble des travaux d'économistes et de sociologues qui cherchent à saisir les déterminants du changement technique et à rendre compte des formes prises par l'innovation.

\section{LES MODĖLES GLOBAUX}

Trois auteurs, Leroi-Gourhan, Mumford et Marx, sont présentés ici qui, au delà de ce qui les rassemble, se distinguent par la définition qu'ils accordent à la technique: pour LeroiGourhan, la technique doit être considérée dans la continuité par rapport à l'évolution naturelle, évolution qu'elle vient prolonger sous une forme extra-corporelle; pour Mumford, la technique se présente comme la continuation de la politique, au sens large, par d'autres moyens, alors que chez Marx, elle se situe dans une dynamique socio-économique.

\section{LEROI-GOURHAN, OU LA TECHNIQUE DANS LE PROLONGEMENT DE L'ÉVOLUTION NATURELLE}

Préhistorien et anthropologue, Leroi-Gourhan a cherché à construire un cadre théorique qui permette à la fois de penser le développement humain dans la continuité de l'évolution du règne animal et de rendre compte de la spécificité humaine définie par l'émergence de sociétés de plus en complexes, accompagnée par la sophistication toujours croissante des techniques. L'évolution naturelle peut être résumée, pour Leroi-Gourhan, par la spécialisation progressive, et inégale selon les espèces, de deux zones corporelles, la face et les membres antérieurs: dans ce cadre, l'homme est envisagé comme le point d'aboutissement de ces deux tendances poussées à l'extrême. L'accès des anthropoïdes à la station debout joue un rôle fondamental dans le processus: la bipédie permet la libération de la main des contraintes de la locomotion, libération qui, elle-même, dégage la face des tâches liées à la recherche de nourriture; par ailleurs, elle entraîne des modifications substancielles dans la manière dont la tête est suspendue au tronc, ce qui autorise la transformation de la boîte cranienne et l'expansion volumétrique du cerveau. L'évolution de la main va être prolongée par l'outil, considéré comme une sécrétion du corps de l'Anthropien, qui, conjoint aux possibilités mécaniques offertes par la bipédie, permet le développement du cerveau ainsi que sa progressive spécialisation, dont la croissance des centres afférents au langage est l'une des expressions. Une fois que l'homme a atteint sa morphologie actuelle, l'évolution va se continuer de façon extra-corporelle au travers du développement conjoint des techniques et de l'organisation sociale, considérée comme une sorte de méta-organisme par rapport à l'organisme individuel. A toutes les étapes, l'évolution se présente comme un jeu dialectique entre deux termes, membre antérieur/ face, outil/cerveau (langage), techniques/société, jeu dont l'issue est commandée par la sélection opérée sous la pression du milieu. L'excorporation progressive, à la fois dans le corps social et dans les techniques, de fonctions auparavant assumées par l'organisme biologique individuel s'accompagne d'une redéfinition des compétences globales du système formé par l'homme et ses appendices, redéfinition qui, elle-même, réouvre les possibilités de l'évolution. Cette théorie débouche sur la constitution d'une techno-logie (science des techniques), envisagée comme le prolongement de la biologie, et qui considère l'outil comme l'équivalent du squelette des paléontologues, à savoir un témoin des rapports entre les êtres vivants et le milieu. 


\section{MUMFORD, LA TECHNIQUE DANS LE PROLONGEMENT DE L'ORGANISATION POLITIQUE}

Le point de départ de la théorie de Mumford est à peu près à l'opposé de celui de LeroiGourhan. Alors que chez ce dernier, il y a continuité de l'évolution humaine avec l'évolution animale et que les possibilités intellectuelles et communicationnelles de l'homme sont le résultat du jeu, sous pression du milieu, de contraintes biologiques et mécaniques, Mumford caractérise d'emblée l'homme par la taille et la puissance de son cerveau: ce sont elles qui lui confèrent dès l'origine une capacité incomparable de symbolisation, capacité qui donnera lieu au développement du langage et des modèles intellectuels. Dans ce cadre, le langage est considéré comme la première, dans le temps et en importance, de toutes les technologies ${ }^{6}$ : c'est l'artefact le plus transportable qui existe et le seul qui permette une totale symétrie entre producteur et consommateur; sa mobilisation est immédiate et sa flexibilité parfaite; bien que constitué d'éléments standardisés, il permet un maximum de variété, d'individualité et d'autonomie. Grâce à son cerveau et au langage, l'homme va développer à la fois des dispositifs techniques qui, comme l'arc et la flèche, ne sont pas de serviles copies de la nature et prouvent le primat des capacités intellectuelles, et des formes d'activité en commun, comme la chasse, qui débouchent sur des organisations sociales plus étendues... jusqu'aux grandes civilisations comme celles de l'Egypte antique. Celles-ci, en rupture avec la civilisation villageoise qui les précède et dont elles sont issues, sont marquées par la découverte d'un nouveau genre d'organisation sociale, ce que Mumford appelle la Mégamachine et dont la puissance est symbolisée par l'érection des pyramides égyptiennes. L'alliance entre le chef de chasse et le prêtre, entre pouvoir temporel et pouvoir spirituel, et l'agrégation, sous leur double houlette, de petites communautés villageoises autonomes - ce qui permet de dégager des surplus rendant possible l'entretien d'une armée, d'une bureaucratie et d'un clergé - sont à l'origine de cette Méga-machine. Celle-ci est caractérisée par une forme d'organisation politico-sociale hypercentralisée qui transforme les hommes en rouages et débouche sur la concentration des forces mécaniques, laquelle est la clé de la puissance et du pouvoir. Partant de là, l'évolution des sociétés peut se comprendre comme le perfectionnement continu de cette Méga-machine, grâce au développement des sciences et des techniques qui permettent de remplacer certains éléments humains de la Mégamachine par des éléments non-humains. Dans cette course à la puissance qui, pour Mumford, ne peut que déboucher sur une catastrophe, tout est inversé dans l'ordre des valeurs: Galilée, qui permet la séparation des sphères subjective et objective, Descartes, qui place l'homme au centre de la création et en fait le maître de la nature via la connaissance, Hobbes qui théorise la Méga-machine et qui fait de l'autorité centralisée la seule planche de salut pour la société, Bacon qui relie science et applications techniques et affirme l'identité entre connaissance et pouvoir, tous ces habituels héros positifs de la civilisation se transforment en criminels les plus odieux pour avoir contribué à la stabilisation et à la croissance de la Méga-machine, au travers de sa matérialisation, rendue possible par l'essor des techniques.

\footnotetext{
${ }^{6}$ GOODY (1979) développe un argument semblable sur l'écriture.
} 


\section{MARX, LA TECHNIQUE DANS LA DYNAMIQUE SOCIO-ÉCONOMIQUE}

On assiste chez Marx à un mouvement analogue de substitution progressive des machines aux hommes, substitution indissociable de l'essor du capitalisme et qui concourt à la concentration du pouvoir socio-économique entre les mains des capitalistes. Certains auteurs considèrent que Marx est le premier sociologue des techniques ${ }^{7}$. Bien que sa "sociologie des techniques" ne soit pas détachable de l'ensemble de sa théorie, nous ne pouvons revenir ici sur sa théorie des échanges ni sur le problème de la plus-value. Nous partirons du point suivant: la différence (ou plus-value) qui existe entre le prix-valeur auquel le capitaliste cède la marchandise produite et la somme des valeurs des éléments (matières premières, énergie, équipements, force de travail) qui ont permis de la produire provient du fait que le salaire versé à l'ouvrier permet au capitaliste d'acheter sa force de travail et non son travail. Autrement dit, la force de travail produit plus de travail que le travail nécessaire à sa reproduction. Ce qui signifie que la journée de travail de l'ouvrier peut être scindée en deux parties, l'une qui sert à la reproduction de la force de travail et l'autre qui représente un surtravail approprié par le capitaliste. Notons qu'un tel montage n'est possible que s'il existe au départ un marché du travail déséquilibré avec, d'un côté, des travailleurs qui n'ont que leur force de travail à troquer pour survivre et de l'autre des capitalistes susceptibles d'acheter cette force de travail.

L'intérêt du capitaliste est d'augmenter la plus-value, ce qui peut se faire soit en allongeant la journée de travail, ce qui revient à augmenter de façon absolue la quantité de surtravail, soit en augmentant la "productivité", c'est-à-dire en augmentant de façon relative la quantité de surtravail par rapport au travail total. Cette augmentation de la productivité peut être obtenue par l'intensification des cadences ou par l'introduction de machines. Mais l'allongement de la journée de travail et l'intensification des cadences se heurtent assez rapidement à des obstacles physiques et sociaux. D'où l'intérêt pour le capitaliste de recourir à la mécanisation de la production. II est très important de noter que l'avantage relatif retiré par un capitaliste d'une augmentation de productivité est transitoire, ce qui confère un caractère dynamique au modèle de Marx: en effet, les marchandises s'échangent à un prix qui correspond à leur "valeur sociale", c'est-à-dire proportionnel à la quantité moyenne de travail incorporé; le capitaliste qui mécanise la production bénéficiera d'une plus-value relative tant que les autres producteurs n'auront pas atteint le même niveau de productivité. Mais, si l'on suppose que tous les capitalistes sont conscients de leur intérêt, le modèle tend vers une homogénéisation de la productivité qui se traduit par une augmentation de la quantité de biens produits et par la baisse de leur prix unitaire (puisque les prix s'établissent à la valeur des produits), ce qui, finalement, est susceptible de bénéficier à l'ensemble de la société sous la forme d'un accroissement général de bien-être. Ce phénomène permet d'expliquer une partie des sentiments, ambivalents, que Marx nourrit à l'égard du machinisme: c'est l'utilisation capitaliste des machines qui est en cause et non les machines elles-mêmes.

Pour comprendre ce que sont les machines dans la pensée de Marx, il est nécessaire de revenir sur l'ensemble du processus historique qui conduit, depuis le Moyen-Age marqué par la prééminence des métiers, à la grande industrie du 19ème siècle. Trois étapes peuvent

\footnotetext{
${ }^{7}$ c'est en particulier le cas de MACKENZIE (1984) et WESTRUM (1991)
} 
être distinguées, caractérisées par la forme d'organisation du travail qui s'y met en place, à savoir: la coopération, la division du travail et le machinisme.

La coopération consiste à réunir dans un même atelier un grand nombre d'ouvriers, ce qui permet: 1) une économie des moyens de production (indépendante des ouvriers), 2) l'émergence du "travail social", c'est-à-dire du travail moyen qui sert à la fixation de la valeur, 3) une émulation et une coordination entre les ouvriers qui entraînent une meilleure efficacité.

Cette première recomposition économique et sociale de l'atelier, qui résulte simplement de la mise en commun des ouvriers, se poursuit dans le passage à la manufacture, caractérisé par une division poussée du travail. Le rassemblement de métiers divers dans un même atelier et la coopération entre des ouvriers de même métier permet une décomposition des tâches en opérations parcellaires et donne naissance à l'ouvrier parcellaire qui "transforme son corps tout entier en organe exclusif et automatique de la seule et même opération simple". Les effets de cette division du travail sont multiples: des gains de productivité sont obtenus par la spécialisation conjointe des ouvriers et des instruments de travail; une classe d'ouvriers déqualifiés apparaît; le "travail social" devient une mesure interne à la manufacture, ce qui permet de substituer à la hiérarchie des métiers une qualification "morale" portée sur l'habileté de l'ouvrier. La manufacture, sous sa forme parfaite, crée un travailleur collectif dont les organes sont constitués par les travailleurs individuels: elle apparaît donc comme une sorte de machinerie socio-technique qui associe forme d'organisation du travail et redéfinition des outils. Cette "rationalisation" du travail productif rend possible le remplacement progressif de l'ouvrier par des mécanismes qui actionnent à sa place les outils, ce qui, pour Marx, suffit à définir la machine: Mackenzie note qu'au travers de cette définition, Marx positionne le développement des machines dans un processus historique et social, ce à quoi l'on peut rajouter que, pour Marx, les grandes mutations socio-économiques intervenues entre le Moyen-Age et le 19ème siècle peuvent être décrites sous la forme d'une redistribution des compétences entre individu, groupe, outils et machines.

La mécanisation des activités productives commence par la mise en place de machinesoutils identiques, souvent mues par une force unique, - on se trouve ici dans un modèle de coopération - et aboutit à la formation de systèmes complexes qui comprennent plusieurs machines aux fonctions et outils différents et qui fonctionnent comme un méga-organisme. Cette dernière étape conduit à l'objectivation de la division du travail instaurée par la manufacture et prolongée par l'assemblage des machines, objectivation qui se traduit par la déqualification totale des ouvriers, agis par les machines, et le transfert de leurs compétences dans les machines, ce qui asseoit la domination du capitaliste sur des bases toujours plus solides. En particulier, le machinisme se satisfait d'une main-d'oeuvre moins pourvue de force (femmes, enfants), ce qui conduit à redéfinir à la baisse la valeur de la force de travail, dorénavant répartie sur davantage de têtes. Une fois le processus de mécanisation entamé, la logique de la plus-value, telle que nous avons explicitée plus haut, 
entraîne une extension progressive du machinisme dans une même branche et de branche à branche ${ }^{8}$.

Comme chez Mumford, les machines et plus généralement les techniques doivent se comprendre comme la matérialisation et l'objectivation d'une organisation sociale qui, chez Marx, se trouve elle-même transformée en retour par les machines. II n'y a donc pas, et cela est valable pour les trois auteurs que nous avons étudiés, de déterminisme à sens unique, de la technique vers le social ou du social vers la technique, mais des séries d'interactions et de rétro-actions entre l'un et l'autre qui les redéfinissent conjointement. Par ailleurs, bien que, à l'instar du sens commun, ces deux entités soient clairement distinguées, elles sont, chez nos trois auteurs, comme organiquement liées à l'intérieur d'une méta-construction qui les dépasse. En d'autres termes, il n'y a pas de différence de nature entre elles (au prix, il est vrai, d'une certaine "technicisation" du social), mais des différences de degré dans la solidité, la prévisibilité et "l'objectivité" des assemblages qui les constituent; on verra que, de ce point de vue, il existe un certain parallèle entre ces différents modèles et ceux qui émergent des théories récentes, bien que, comme précédemment, les opérations de passage d'un registre à l'autre soient non problématiques.

L'œuvre de Marx, en ce qui concerne plus spécifiquement le problème des techniques, a eu plusieurs postérités: dans les travaux de certains critiques des technologies, comme Marcuse, dans ceux d'une partie de la sociologie du travail que nous laisserons de côté ici, mais dont on peut dire qu'elle s'est organisée autour des problèmes de qualification/ déqualification entraînés par la mécanisation puis l'automatisation des activités de production, et enfin, dans un certain nombre de travaux "féministes", que nous aborderons ici rapidement.

Dans les premiers travaux de Cynthia Cockburn par exemple, l'évolution technique dans l'univers productif peut être comprise comme le résultat de la combinaison de deux facteurs, à savoir: dans la perspective marxiste, la recherche par le capitaliste d'un plus grand asservissement des travailleurs au travers de la mise en place d'un certain nombre de dispositifs techniques qui permettent d'accroître la productivité et d'instrumentaliser la main d'oeuvre en la dépossédant de ses compétences, et d'autre part, la recherche, plus ou moins consciente, par les hommes du maintien du rôle subalterne des femmes en liant, toujours au travers des caractéristiques des dispositifs techniques, compétences techniques et compétences "masculines". Le raisonnement s'appuie sur deux formes de causalité, qui permettent une circulation dans les deux sens entre le technique et le social, et se bouclant sur lui-même, installe des mécanismes d'auto-renforcement de ces deux tendances: dans nos sociétés, la différenciation sexuelle se construit, dans toutes les occasions de la vie quotidienne, par l'attribution et l'incorporation de compétences spécifiques aux hommes d'un côté (force physique, efficacité technique, puissance intellectuelle) et aux femmes de l'autre (qui se définissent en négatif par rapport à ces caractéristiques, et en positif par des aptitudes concernant la vie familiale et domestique); cette différenciation primaire est réinscrite dans les dispositifs techniques de l'univers productif, inscription qui permet que les

\footnotetext{
${ }^{8}$ II faut bien entendu que la quantité de travail incorporée dans la machine ne soit pas trop importante, ce qui suppose rapidement une mécanisation de la production des machines.
} 
femmes soient écartées des métiers qualifiés et restent subordonnées aux hommes. Elle est rendue possible par le fait que, pour les mêmes raisons, ceux qui conçoivent les dispositifs techniques sont des hommes; et elle est ensuite renforcée par un certain nombre d'institutions, dont les syndicats, qui protègent les professions masculines de l'invasion féminine.

Ce type d'analyse opère un glissement significatif par rapport aux théories précédemment présentées: le processus de conception des dispositifs techniques y est réintroduit comme une opération à part entière et ne se réduit plus à la translation pure et simple du social vers la technique. Se trouve ainsi ouverte la possibilité d'une analyse constructiviste de la technologie qui redonne, au moins en théorie, leurs chances à une pluralité de trajectoires pour le développement technologique.

\section{LES MODĖLES À CAUSALITÉ LOCALE}

Les modèles regroupés dans cette partie se distinguent des précédents par leur rejet de toute grande cause générale permettant d'expliquer la direction et la forme prise par le développement des technologies. Plus précisément, on observe, chez les économistes comme chez les sociologues, la recherche d'une troisième voie qui permette de sortir des alternatives posées par leurs prédécesseurs, à savoir chez les économistes l'alternative entre "demand pull" et "supply push", et chez les sociologues, celle qui oppose le "social determinism" au "technical determinism".

\section{GILFILLAN/ OGBURN OU LA DOUBLE DÉTERMINATION}

Avant d'entrer dans le détail de ces travaux récents, nous voudrions revenir sur I' "Ogburn generation"9, constituée d'un groupe de chercheurs qui sont considérés comme les pionniers de la sociologie des techniques et qui ont, les premiers peut-être de façon aussi explicite, posé l'existence d'une double détermination des techniques par le social et du social par les techniques. Pour Ogburn, les objets techniques sont essentiellement définis par ce qu'ils font, par leurs "fonctions", ce qui revient à postuler d'emblée la nature sociale des techniques, hypothèse que renforce l'idée selon laquelle, au stade de la conception, la "situation sociologique" représente l'un des facteurs explicatifs les plus importants. Si Ogburn apparaît comme le chef de file du mouvement, c'est essentiellement Gilfillan qui a développé une théorie de l'invention, Ogburn lui-même s'intéressant davantage à l'impact des techniques qu'à leur émergence. Pour Gilfillan, l'invention est un processus continu qui procède de l'accumulation de changements de faible amplitude, et peut être décrite comme une combinaison nouvelle entre des éléments pour la plupart pré-existants; ces éléments sont très divers et comprennent aussi bien des dispositifs techniques, que les modes de conduite de ces dispositifs, les matériaux et méthodes employés pour les construire, les matières premières nécessaires à leur fonctionnement, l'accumulation de capital qu'ils supposent, les savoir-faire et compétences qu'ils réclament, la façon dont ils sont reliés à d'autres aspects de la vie sociale et économique, etc. Les causes de l'invention sont avant

\footnotetext{
${ }^{9}$ Selon la dénomination de Ron WESTRUM (1991)
} 
tout sociales - le progrès scientifique est rarement à l'origine des inventions; et même, il arrive souvent que les inventions précèdent le développement des sciences qui leur sont associées - et elles doivent être recherchées à deux niveaux, un niveau microsociologique l'origine, la culture, les aspirations de tous ceux qui mettent en forme l'invention, à savoir principalement les inventeurs eux-mêmes et les entrepreneurs - et un niveau macrosociologique qui comprend aussi bien des facteurs économiques, culturels que démographiques. L'appréhension de l'évolution de ces facteurs qui génèrent de nouveaux besoins peut se faire soit par la variation d'un certain nombre d'indicateurs dont le prix est l'un des plus importants, soit par l'intermédiaire de relais humains, qui vont des inventeurs eux-mêmes au grand public en passant par une "intelligent and technologicly minded class". Ce processus étant avant tout social, les individus y ont une place des plus restreintes: comme chez Gille, l'invention naît de la rencontre entre des besoins et des ressources techniques, scientifiques et économiques, ce qui implique que certaines inventions, dont le principe est posé avant "l'heure", ne soient développées que plus tardivement, ou qu'une même invention soit mise au point simultanément par plusieurs inventeurs. Une fois que les inventions sont diffusées, elles deviennent elles-mêmes le moteur de changements sociaux, phénomène qui intéresse plus particulièrement Ogburn. Concilier une théorie qui se propose de montrer comment des entités inanimées comme les objets induisent des changements sociaux avec une philosophie qui dote l'individu d'une liberté et d'une capacité de choix est l'un des problèmes principaux que va essayer de résoudre Ogburn. Pour ce faire, il se donne d'abord une théorie de la cause, calquée sur le modèle scientifique: pour que l'on puisse parler de deux phénomènes en termes de cause et d'effet, il faut qu'ils varient de façon concommitante et que l'on puisse les relier par une chaine de médiateurs. Or, à moins de se situer sur des échelles de temps considérables, la psychologie, l'intelligence, les aptitudes individuelles sont, pour Ogburn, des variables statistiques mais non historiques. Ce qui l'amène à considérer qu'une phrase comme "les automobiles ont causé le développement des motels" est pleinement dotée de sens, alors qu'il dénie toute vertu explicative à un énoncé du type "Denis Papin a inventé la machine à vapeur". Mais, pour que l'automobile puisse accéder au rang de cause, il faut que suffisamment d'individus aient choisi d'utiliser ce nouveau moyen de locomotion, choix qu'ils font, en principe au moins, librement en fonction de leurs besoins, lesquels ne sont pas nécessairement en parfaite correspondance avec ceux qui ont été identifiés par les inventeurs; de plus, l'utilisation qu'ils font de tel ou tel dispositif technique n'est pas entièrement programmée de l'intérieur par la conception des dispositifs - il existe une certaine flexibilité des objets - ces deux réserves permettant à Ogburn d'éviter le piège du "déterminisme technique". De la sorte, il peut affirmer avoir montré "how inanimate objects such as inventions can cause social changes through the media of active human agents with capacity of choices". Partant de là, l'impact social des technologies peut être appréhendé par des méthodes statistiques qui permettent de mettre en rapport la diffusion d'une technologie avec un certain nombre de phénomènes sociaux.

Bien que l'on puisse tracer certains parallèles entre les théories de l'”Ogburn generation" et les théories plus récentes auxquelles nous allons maintenant nous intéresser - le refus d'un modèle linéaire allant de la science vers les applications et le marché, le rejet d'un déterminisme technique, la technique comme ensemble complexe de dispositifs, savoirs, savoir-faire, la présence d'acteurs aux motivations hétérogènes - plusieurs autres éléments nous paraissent aller plutôt dans le sens d'une divergence: d'une part, Ogburn et ses 
collègues s'appuient sur une coupure radicale entre tout ce qui concerne d'un côté la conception des innovations et de l'autre leur diffusion, la médiation entre ces deux termes étant assurée par des "besoins" pré-existants à l'innovation; d'autre part, la technique ellemême est finalement dotée de peu d'épaisseur et, bien que le modèle boucle sur lui-même, en dernier ressort, ce sont quand même les facteurs sociaux qui constituent le moteur de l'innovation.

\section{L'INNOVATION COMME PROCESSUS SOCIAL}

Nous organiserons ici notre présentation autour de trois groupes d'auteurs, les économistes Nelson \& Winter, Dosi, et les sociologues Pinch \& Bijker. Les similitudes entre leurs travaux nous sont apparues suffisamment fortes pour que l'on puisse, au delà des barrières disciplinaires et des différences de vocabulaire, tenter une présentation conjointe de leurs modèles; ces similitudes ne sont pas le simple fruit du hasard: les uns connaissent plus ou moins ce que font les autres; comme nous l'avons dit plus haut, la quête d'une solution pour sortir de l'alternative binaire demand/supply ou social determinism/technical determinism tend leur réflexion dans des directions convergentes; enfin, la sociologie des sciences a constitué une source d'inspiration commune - même si les auteurs considérés ne sont pas tout à fait les mêmes - aux travaux de Dosi et de Pinch \& Bijker.

Chez ces trois auteurs, la technologie est définie comme un ensemble complexe qui comprend et associe des dispositifs techniques, des savoirs et des savoir-faire, une certaine appréhension de l'état de l'art et des problèmes qui restent posés, une représentation des méthodes disponibles ou pertinentes pour résoudre ces problèmes, un certain nombre d'idées sur les leçons à tirer des expériences passées et sur l'avenir possible ou probable des technologies. La technologie se présente donc essentiellement comme du savoir, qui peut être "embodied" dans des artefacts, ou "disembodied" par rapport aux artefacts, mais "embodied" dans la pratique quotidienne de tous les acteurs concernés par le développement technologique. Ce savoir peut être propre à un groupe d'acteurs (Pinch \& Bijker), une firme ou un secteur industriel (Nelson \& Winter), ou avoir une portée plus générale, comme chez Dosi où il prend la forme de "paradigmes technologiques".

Les acteurs impliqués, toujours humains et souvent formés de collectifs, sont dans ces trois modèles très divers ${ }^{10}$ : on voit intervenir aussi bien les entreprises que les ingénieurs ou les chercheurs, les politiques, les organismes publics dans tous les domaines (recherche, industrie, mais aussi éducation, santé etc.), les "utilisateurs" primaires (compagnies aériennes dans le cas des avions par exemple) et secondaires (passagers de ces compagnies aériennes); de plus, chaque acteur a sa propre définition de ce qui est "profitable", ou autrement dit des objectifs généraux qu'il s'assigne et qui peuvent être le profit économique pour une entreprise privée, mais aussi une meilleure qualité de soins pour le secteur médical ou une meilleure capacité de défense pour les organisations militaires. Pinch \& Bijker insistent sur le fait que la liste des acteurs concernés, ce qu'ils appelent les "relevant social groups", est spécifique de chaque innovation et revêt un caractère

\footnotetext{
${ }^{10}$ dans cette lignée de travaux, on pourra voir ALLEN (1983) et VON HIPPEL $(1982,1986)$
} 
stratégique dans le développement des innovations: ce sont eux qui définissent les problèmes auxquels l'innovation doit apporter une réponse.

Chez Nelson \& Winter, les stratégies de R\&D qui donnent naissance aux innovations sont le résultat d'un processus heuristique, propre à chaque entreprise, qui doit être conçu comme un ensemble de procédures permettant de définir des objectifs et des méthodes pour les atteindre. L'heuristique incorpore non seulement des savoirs proprement technologiques mais aussi des modèles d'organisation interne, des méthodes d'appréhension et de prise en compte de la demande etc. Parmi les différentes stratégies qui émergent de ces processus heuristiques, certaines ont la particularité d'apparaître comme souhaitables ou rentables sous une large gamme de conditions du côté de la demande: elles représentent ce que Nelson \& Winter appelent des "trajectoires naturelles" et regroupent par exemple les stratégies qui visent à l'exploitation des économies d'échelle, à la mécanisation des opérations de production, ou à l'incorporation de dispositifs électroniques. Situation très semblable à celle qui est décrite par Dosi lorsque l'on se trouve à un moment où un paradigme est solidement établi: dans ce cas-là, en effet, les directions dans lesquelles se portent les efforts de développement sont fortement pré-déterminées par les conceptions que se font a priori les acteurs sur les problèmes à résoudre et les méthodes qui doivent être utilisées à cette fin. En revanche, et à la différence de Nelson \& Winter qui conçoivent le développement technologique comme un processus plutôt continu, l'instauration d'un nouveau paradigme suppose une remise en cause à la fois de la technologie - on se situe dans des domaines où les savoirs sont encore incertains - et du marché - il s'agit alors de construire presque ex nihilo une demande pour des produits qui n'ont pas d'équivalents dans le passé. Dans les deux modèles cependant, la progressive détermination des trajectoires technologiques s'effectue par un processus de sélection. Chez Nelson \& Winter, l'environnement sélectif comprend tous les acteurs extérieurs à l'organisation (entreprise ou autre) en charge du développement de l'innovation: parmi toutes les possibilités envisageables aux différents stades de développement, les acteurs vont sélectionner celles qui leur paraissent leur assurer le meilleur profit, quelle que soit par ailleurs la définition qu'ils adoptent de ce profit; dans cette perspective, le marché est seulement l'un des environnements sélectifs possibles, celui auquel jusque là les économistes se sont surtout intéressés. Pour Dosi, la sélection est le résultat de deux mouvements combinés, l'un qui affecte la détermination des mécanismes de génération des mutations - l'instauration d'un paradigme peut être décrite en ces termes - et l'autre qui opère la sélection proprement dite entre les différents "mutants" - le marché en est ici le principal agent.

De façon analogue, les mécanismes de variation-sélection sont au cœur du modèle de Pinch \& Bijker: chaque variante peut être décrite par l'ensemble des problèmes posés - auxquels elle prétend apporter une réponse - et les "relevant social groups" qui les posent, les uns étant définis par les autres. Mais chaque acteur est susceptible d'avoir une interprétation particulière des problèmes et de l'adéquation des solutions qui sont proposées, ce qui crée des situations de controverses. La sélection opérée entre les variantes et la stabilisation progressive de certaines solutions s'interprète dans ce schéma comme la mise en convergence de ces différentes interprétations: à l'issue du processus, chaque "relevant social group" considère que les problèmes sont résolus, ce consensus étant obtenu soit parce que certains acteurs ont la capacité d'imposer une clôture rhétorique de la controverse 
- ils "prétendent" que les problèmes sont résolus - soit parce qu'au cours des négociations qui accompagnent l'innovation, les problèmes ont été collectivement redéfinis.

\section{CONCLUSION}

Se situant dans un cadre évolutionniste, les trois auteurs que nous venons de présenter insistent sur le caractère non linéaire du progrès technique et mettent en avant la pluralité des trajectoires amorcées à un moment donné par l'ensemble des acteurs du changement technique; de ce point de vue, se trouve installée dans les modèles une certaine symétrie entre les innovations qui réussissent et celles qui échouent: d'une part, l'échec n'est plus imputable ni à l'incompétence des innovateurs ni au conservatisme des utilisateurs - ces deux groupes d'acteurs sont dotés d'autant d'intelligence, ils ont simplement des objectifs différents - d'autre part, il est constitutif du processus d'innovation, puisque, par définition, la sélection suppose que certaines solutions soient retenues et d'autres abandonnées. Par ailleurs, ces modèles établissent une continuité entre toutes les étapes de l'innovation, de la conception à la diffusion, essentiellement en faisant remonter dans la conception un certain nombre de facteurs "sociaux". En revanche, même si ces facteurs présentent une diversité et une complexité telles qu'ils génèrent une incertitude irréductible, ils sont supposés préexister au processus d'innovation; tout se passe comme si les innovateurs contribuaient à l'accroissement des connaissances sur la société, mais n'influaient que marginalement sur son devenir. Reconnaissons aussi que ces modèles ont pour objet la compréhension des mécanismes du changement technique et non l'intégration de la technique dans une théorie du changement social. Ceci constitue une différence essentielle entre ces modèles et certains de ceux que nous allons maintenant aborder.

\section{LES MODĖLES DU "SEAMLESS WEB" OU L'ÉMERGENCE DES RÉSEAUX}

La notion de "seamless web" a été proposée par Thomas $\mathrm{P}$. Hughes pour rendre compte de I'hétérogénéité des éléments associés dans ce que Hughes appelle un système technologique et de l'impossibilité qu'il y a à découper dans ce système des pièces d'une texture uniforme, que celle-ci soit sociale, technique, ou économique. Pour Hugues et les auteurs que nous avons réunis ici, les techniques sont à la fois "society shaped" et "society shaping", avec une différence fondamentale par rapport aux modèles que nous avons étudiés dans la deuxième partie: il y a autant d'incertitudes sur la société que sur la technique, ou, autrement dit, l'innovation est un processus de stabilisation conjointe du social et de la technique et aboutit à des arrangements hybrides dans lesquels éléments techniques et éléments sociaux sont indissociablement entremêlés.

\section{DES TECHNIQUES HÉTÉROGĖNES}

Nous avons vu dans la partie précédente qu'un certain nombre d'économistes et de sociologues se sont efforcés de rapprocher l'offre de techniques de la demande: ils ont en particulier montré comment le contexte de l'adoption et de l'utilisation influait sur le contexte 
de la conception et réciproquement. Ici, un pas supplémentaire est franchi dans la mesure où les dispositifs techniques n'ont de sens que rapportés à l'ensemble des éléments auxquels ils sont associés. Cette idée peut être déclinée de nombreuses façons: ainsi, pour l'historien T. Hughes, la réussite du système électrique ne peut s'expliquer que parce que ses promoteurs ont, en même temps qu'ils concevaient les éléments techniques, réorganisé le monde extérieur et que ces deux opérations ont été inextricablement liées l'une à l'autre; la constatation de cette interdépendance entre le dispositif technique et son environnement conduit, pour l'économiste $P$. David, à une critique radicale de la notion de substituabilité des techniques; enfin, Rosenberg abolit définitivement la frontière entre l'offre et la demande en montrant comment le travail de conception et de qualification des techniques se poursuit jusque chez l'utilisateur.

A première vue, le modèle développé par Hughes semble apparenté à celui de Bertrand Gille, à ceci près que Hughes ne suppose pas la séparation et l'autonomie des sphères technique, politique, économique, sociale, etc., ce qui, comme nous allons le voir, a des conséquences considérables sur l'analyse de l'innovation. Pour Hughes, un système technologique, qui se présente comme un ensemble complexe associant connaissances scientifiques, dispositifs techniques, juridiques, politiques, économiques, organisationnels, etc., se définit essentiellement comme une réorganisation du monde physique et social en vue de résoudre des problèmes considérés, au moins par certains acteurs, comme importants. L'imbrication des différents éléments associés dans le système technologique est telle qu'elle suppose un processus de conception collectif qui rassemble et coordonne des individus aux compétences différenciées: ainsi, le financier, le scientifique fondamentaliste ou le "public relation" sont a priori aussi indispensables au développement des innovations que l'ingénieur ou le technicien. La stabilisation du système technologique dépend directement du nombre d'éléments hétérogènes qui le constituent: pour le développement des réseaux électriques qui intéressent Hughes, l'accord des autorités politiques est aussi crucial que la mise au point d'une lampe à incandescence; ceci entraîne certaines conséquences sur la dynamique des systèmes: ainsi, un système de grande taille, qui associe beaucoup d'éléments, acquiert un momentum qui s'oppose à toute transformation radicale. Du fait même de l'interdépendance entre les éléments, la capacité de croissance et d'adaptation du système se trouve limitée: à un moment donné, les transformations opérées sur certains éléments font apparaître l'insuffisance d'autres éléments qui n'ont pu progresser au même rythme. Ces "reverse salient", qu'ils soient organisationnels, techniques ou commerciaux, se transforment une fois perçus en une série de "critical problems": dans certains cas, un simple réaménagement du système existant permet la résolution de ces "critical problems"; en revanche, lorsqu'elle suppose une remise en cause des différents liens qui caractérisent le système technologique, l'on peut assister à l'émergence d'un nouveau système technologique.

A l'instar de Hughes, Paul David a mis en évidence la multiplicité et l'hétérogénéité des éléments associés autour d'une technologie particulière, associations qui sont à l'origine de ce qu'il appelle des "interdépendances techniques": s'élevant contre une description platement fonctionnelle des techniques qui conduit à postuler trop vite leur substituabilité, David montre que l'efficacité et même la possibilité de fonctionnement d'une technologie est subordonnée à une série de conditions sociales, géographiques, juridiques, commerciales, 
etc., qui sont en quelque sorte présupposées par la forme même de la technologie. Ce qui permet d'expliquer les rythmes différents d'adoption des techniques selon les pays ou les régions sans être obligé d'imputer aux uns, un caractère de pionniers, et aux autres, une mentalité rétrograde. A la différence de Hughes, chez qui les utilisateurs finals apparaissent un peu comme le point d'aboutissement naturel des systèmes technologiques, David les installe pratiquement à parité avec les innovateurs: parce que les acteurs sont parfaitement conscients de ce que la validité de leurs choix dépend des choix des autres acteurs, en raison en particulier de la possibilité d'économies d'échelle et de l'existence d'externalités de réseau, leur analyse des interdépendances techniques et l'anticipation qu'ils font des comportements des autres jouent un rôle crucial dans le développement et l'adoption des technologies. Ce qui peut conduire dans certains cas à favoriser durablement une technologie non optimale, du strict point de vue de ces caractéristiques techniques internes, simplement parce que certains éléments intervenus très tôt dans son développement ont enclenché la mécanique performative des anticipations et ont irréversibilisé une série de choix initiaux: ainsi, l'existence précoce de formations à la dactylographie sur clavier QWERTY a suffi à orienter les anticipations et les choix des employeurs et des employés dans la même direction, alors qu'il a été démontré que d'autres dispositions de clavier permettaient une vitesse de frappe bien plus élevée.

Chez Rosenberg, le modèle se complexifie encore dans la mesure où le processus d'innovation ne s'arrête pas à la mise sur le marché des premiers exemplaires d'une technologie, mais se poursuit chez les premiers utilisateurs qui, au travers du "learning by doing", transforment la technologie, définissent son environnement, développent des savoirs et des savoir-faire, tous éléments qui sont aussi importants que le dispositif initial pour assurer la fiabilité et la rentabilité à long terme de la technologie. D'où l'idée que l'adoption d'une technologie dépend des anticipations faites par les acteurs non seulement sur le comportement des autres, mais sur les capacités d'évolution de la technologie en question, des technologies complémentaires qui sont susceptibles d'en améliorer les performances et d'en étendre le champ d'application, et, enfin, des technologies concurrentes, anciennes et nouvelles, qui sont elles aussi capables de redéfinir les termes de comparaison. Ce qui conduit, comme chez Hughes, à considérer que l'innovateur doit se battre autant pour la mise au point des dispositifs techniques que pour emporter la conviction d'éventuels utilisateurs.

\section{LA TECHNIQUE COMME RESEAU}

L'analyse de l'articulation entre ces deux éléments constitue l'un des points d'intérêt majeur pour un ensemble d'auteurs, parmi lesquels on citera Callon, Latour, Law, Mackenzie, qui constitue un courant important de la sociologie récente des techniques. Tout comme dans les modèles que nous venons de présenter, la multiplication et le renforcement des associations entre des éléments hétérogènes est au cœur du processus d'innovation. Cependant, les mécanismes par lesquels un certain nombre d'acteurs sont intéressés au développement d'une innovation ne sont pas disjoints de ceux qui définissent et stabilisent progressivement l'innovation elle-même: par la négociation des contenus techniques qui conduit à leur redéfinition, s'accroît la capacité du dispositif à traduire les besoins, aspirations, objectifs supposés de tous ceux qu'il se doit d'intéresser. Dans cette 
perspective, on ne peut orienter les anticipations des utilisateurs simplement par le recours à des artifices rhétoriques: seule la construction d'un réseau de relations, dans lequel la technique joue un rôle primordial, permet d'augmenter la prévisibilité de chacun des éléments associés et d'irréversibiliser peu à peu les choix passés; en ce sens, les interdépendances techniques de David ou le momentum de Hughes ne sont pas seulement le résultat, plus ou moins désiré et désirable, d'une histoire révolue, mais peuvent être utilisés comme des stratégies permettant d'étendre et de consolider un réseau. Dans ce modèle, l'innovation est donc un processus socio-technique au sens fort du terme, car chaque décision sur les contenus techniques engage une définition de ce que sont les entités (humaines ou non-humaines) intéressées ou à intéresser, des problèmes qui se posent à elles, et de la manière dont on peut les résoudre. Ces décisions ne sont pas prises à l'aveuglette: une part importante du travail de l'innovateur consiste à se donner des représentants ou des porte-parole de ceux qu'il lui faut intéresser; dans cette perspective, le recours à la science doit être interprété comme une des méthodes permettant de se donner des porte-parole d'entités non humaines. L'incertitude inhérente au processus d'innovation et sur laquelle s'accordent la plupart des analystes - prend sa source dans ces procédures de représentation: un simple changement d'échelle peut parfois à bouleverser les conditions de fonctionnement d'un dispositif, le modèle de laboratoire ne suffisant pas à représenter les phénomènes industriels; de même, dans d'autres cas, les revendeurs, censés être au plus près des utilisateurs, s'avèrent incapables de prédire leurs comportements face à un nouveau produit. Les exemples de ces trahisons imprévues sont innombrables, le point important étant ici qu'il n'y a pas dans l'analyse de différence irréductible entre l'enrôlement des humains et celui des non-humains, même si, dans le détail, les méthodes utilisées par les acteurs dans chacune de ces configurations peuvent être assez dissemblables.

Une innovation réussie réalise l'ensemble des hypothèses sur lesquelles son développement s'est appuyé et attribue, en les naturalisant, des propriétés à la fois aux dispositifs et aux humains: c'est ainsi que, dans ce modèle, doit se comprendre le rôle de la technique dans la fabrication de nos sociétés, comme une des modalités par lesquelles se redistribuent de façon plus ou moins stable les compétences des humains entre eux et avec les nonhumains.

\section{CONCLUSION}

Les différents auteurs et courants de recherche que nous avons regroupés dans cette partie présentent un certain nombre de traits communs que nous condenserons dans le terme de "réseau", utilisé comme modalité de description et d'explication: la forme prise par les dispositifs techniques, leur succès ou leur échec, leurs domaines d'utilisation, leurs "impacts", etc., tous ces éléments condensent l'ensemble des liens qui ont été noués, plus ou moins solidement, tout au long du processus d'innovation, entre le dispositif lui-même et les éléments qui constituent activement son environnement. Aucun moteur externe, aucune grande cause univoque ne peut rendre compte du développement technique ou, autrement dit, la différence entre les phénomènes locaux et les phénomènes globaux tient dans l'extension différentielle des réseaux. Alors que le système se présente comme un assemblage d'éléments plutôt passifs qui, à partir d'une perturbation externe, réagissent de façon mécanique les uns sur les autres, le réseau est constitué d'éléments dont le degré de 
passivité/activité est susceptible de varier au cours du temps, et qui sont dotés a priori d'une capacité de recomposition des liens qui les attachent les uns aux autres et par lesquels ils s'entre-définissent; l'innovation, en tant que dispositif technique d'une forme particulière, circulant dans un espace spécifique, est donc le résultat d'un processus éminemment collectif, la définition et l'extension de ce collectif variant selon les auteurs. Enfin, ni la technique, ni le social ne jouissent d'une position d'extériorité par rapport au réseau, ce qui interdit de se poser la question du déterminisme de l'un par l'autre: l'innovation est l'une des modalités par lesquelles les compétences sont redistribuées entre les différentes entités qui peuplent le monde, humaines et non-humaines, techniques et sociales.

\section{CONCLUSION}

La forme que nous avons donnée à la présentation précédente avait pour fonction d'éviter deux écueils classiques dans ce type d'exercice: une tendance critique qui peut conduire à une présentation superficielle, trop prompte à reprocher aux auteurs de ne pas avoir répondu à des questions qu'ils ne se sont pas posés, ou une tendance positiviste qui conçoit, au moins le temps de la présentation, l'évolution d'une discipline comme un processus cumulatif. Dans ces deux cas, un champ de recherches est défini comme un espace homogène de questions et de problèmes: ce parti de présentation, acceptable dans le cas de domaines fortement structurés, ayant derrière eux une longue histoire de débats organisés, devient difficile à tenir lorsque l'on a affaire, comme en sociologie des techniques, à une discipline constituée récemment... autour d'un objet, les techniques, qui ont depuis fort longtemps inspiré réflexions, commentaires et analyses de la part de chercheurs provenant des horizons les plus divers. Si l'on voulait définir la spécificité de ce courant de recherches, c'est peut-être sur ce dernier point qu'il conviendrait d'insister: la sociologie des techniques représente le lieu duquel l'on peut embraser ce vaste paysage des sciences humaines, luimême réorganisé par l'axe que définit notre question de départ: genèse technique de la sociétél genèse sociale des techniques? Que l'on y croise l'économie, la philosophie, l'anthropologie etc. ne doit pas nous surprendre: car l'ambition même des recherches actuelles est précisément, en ignorant les barrières disciplinaires, de proposer un cadre d'analyse général qui réintègre dans les sciences humaines non seulement les techniques, mais toute la cohorte de non-humains qui constituent, au même titre que les humains, notre espace social. En d'autres termes, c'est à une redéfinition même de la sociologie que nous sommes ici invités. 


\section{REFERENCES}

ALLEN, R. C. 1983. "Collective invention" Journal of Economic Behavior \& Organization n4: p. 124.

BIJKER, W. E., HUGHES, T. P. and T. PINCH, 1989. The Social Construction of Technological Systems, Cambridge - London: The MIT Press.

CALLON, M. 1981. " Pour une sociologie des controverses technologiques" Fundamenta Scientiae 2, 3/4: 381-399.

CALLON, M. 1986. "Éléments pour une sociologie de la traduction : la domestication des coquilles St-Jacques et des marins pêcheurs dans la baie de St. Brieuc", L'Année Sociologique, numéro spécial La sociologie des Sciences et des Techniques, vol. 36, p 169-208.

CALLON, M. 1989. "Réseaux technico-économiques et irréversibilité", in Figures de l'irréversibilité en économie, Robert BOYER (dir.), Paris: Ed. EHESS.

CALLON, M. 1993. Four models for the dynamics of science. In Science, Technology and Society Handbook, Los Angeles: Sage.

COCKBURN, C. 1981. "The Material of Male Power" Feminist Review 9: 41-58.

DAVID, P. A. 1986. "Understanding the Economics of QWERTY: the Necessity of History" In Economic History and the modern Economist., W. N. PARKER (ed), Oxford: Basil Blackwell

DAVID, P. A. 1986. "La moissonneuse et le robot. La diffusion des innovations fondées sur la micro-électronique" In Les enjeux du changement technologique, J.-J. SALOMON and G. SCHMEDER (eds), Paris: CPE-Economica

DOSI, G. 1982. "Technological paradigms and technological trajectories. A suggested interpretation of the determinants and directions of technical change." Research Policy 11, 147-162.

ELLUL, J. 1977. Le système technicien. Paris: Calmann-Lévy.

GILFILLAN, S. C. 1963. The sociology of invention. Cambridge: MIT Press.

GILLE, B. 1978. Histoire des techniques. Paris: Gallimard, La Pléïade. 
GOODY J., 1979. Raison (la) graphique:la domestication de la pensée sauvage, Paris : Ed. de minuit.

HUGHES, T. P. 1983. Networks of power. Electrification in Western Society,1880-1930. Baltimore:The Johns Hopkins University Press.

HUGHES, T. P. 1989. "The Evolution of Large Technological Systems" In BIJKER, HUGHES and PINCH (eds), p.51-82.

LAFITTE, J. 1972. Reflexions sur la science des machines (nouv. ed.). Paris : J. Vrin.

LATOUR, B. 1984. Les Microbes: guerre et paix, suivi de Irréductions. A.-M. Métaillé, collection Pandore.

LATOUR, B. et WOOLGAR, S. 1988. La Vie de laboratoire- La production des faits scientifiques. La Découverte. Version anglaise: 1979. Laboratory Life : the Social Construction of Scientific Facts, Los Angeles/Londres: Sage.

LATOUR, B. 1989. La Science en action. Editions La Découverte, Paris. Version anglaise: 1987. Science in Action, How to Follow Scientists and Engineers through Society, Harvard University Press (U.S.): Open University Press (G.B.).

LATOUR, B. 1992. Aramis, ou l'amour des techniques, Paris: La Découverte.

LAW, J. 1989. Technology and Heterogeneous Engineering: The Case of the Portuguese Expansion. In BIJKER, HUGHES and PINCH (eds), p. 111-134.

LEROI-GOURHAN, A. 1964. Le geste et la parole. 1, Technique et langage. 2, La mémoire et les rythmes. Paris: Albin Michel.

MACKENZIE D., 1984. "Marx and the Machine", Technology and Culture, 25-3, 473-502.

MACKENZIE, D. and WAJCMAN, J. (eds) 1985. The Social Shaping of Technology. Milton Keynes: Open University Press.

MACKENZIE, D. 1988. "Stellar-inertial guidance: a study in the sociology of military technology" Science, Technology and the Military XII: 187-241.

MACKENZIE, D. 1990. Inventing accuracy. A historical Sociology of Nuclear Missile Guidance. MIT Press. 
MARCUSE, H. 1968. L'homme unidimensionnel. Essai sur l'idéologie de la société industrielle avancée. Paris: Editions de Minuit.

MARX, K. 1969. Le capital. Paris: Garnier Flammarion.

MUMFORD, L. 1973. Le mythe de la machine. 1, La technologie et le développement humain. 2, Le pentagone de la puissance. Paris: Fayard.

NELSON, R. R. and WINTER, S. G. 1977. "In search of a useful theory of innovation", Research Policy 6: p. 36-76.

NELSON, R. and WINTER, S. G. 1982. An evolutionnary theory of economic change. Belknap: Harvard University Press.

OGBURN W. F. 1957. "How technology causes social change" in Technology and social change, edited by ALLEN F. R., HART H., MILLER D. C., OGBURN W. F. et NIMKOFF M. F., New York: Appleton-Centurey-Crofts.

PINCH, T. and W. BIJKER. 1989. "The Social Construction of Facts and Artifacts: Or How the Sociology of Science and the Sociology of Technology Might Benefit Each Other" in BIJKER, HUGHES and PINCH (1989) 532.

ROSENBERG, N. 1976. "On technological expectations" The Economic Journal 86, sept.: 523-

ROSENBERG, N. 1985. Inside the Black Box. Technology and Economics. Cambridge: Cambridge University Press.

ROSENBERG. 1976. Perspectives on Technical Change. Cambridge: Cambridge University Press.

SIMONDON, G. 1958. Du mode d'existence des objets techniques. Paris, Aubier.

VON HIPPEL, E. 1976. "The Dominant Role of Users in the Scientific Instrument Innovation Process" Research Policy 5: p. 212-239.

VON HIPPEL, E. 1982. "Appropriability of innovation benefit as a predictor of the source of innovation" Research Policy 11, 95-115. 
WESTRUM, R. 1991. Technologies and society, Belmont: Wadsworth.

WINNER, L. 1985 "Do artefacts have politics?" in The Social Shaping of Technology: How the Refrigerator got its Hum., edited by D. MACKENZIE, et J. WAJCMAN, Milton keynes : Open University.

WINNER, L. 1983. Autonomous technology. Technics-out-of control as a theme in political thought., Cambridge: MIT Press. 